\title{
Integrative Chinese and Western Medicine for patients after Percutaneous Coronary Intervention (ICWM-PCI): A Prospective Observational Real- World Cohort Study
}

\section{Siyu YAN}

Chinese Academy of Medical Sciences \& Peking Union Medical College Fuwai Hospital

\section{Wei-Xian Yang}

Chinese Academy of Medical Sciences \& Peking Union Medical College Fuwai Hospital

Pei-Pei Lu

Chinese Academy of Medical Sciences \& Peking Union Medical College Fuwai Hospital

\section{Xuan-Tong Guo}

Chinese Academy of Medical Sciences \& Peking Union Medical College Fuwai Hospital

\section{Cai-Xia Guo}

Chinese Academy of Medical Sciences \& Peking Union Medical College Fuwai Hospital

\section{Yan-Ni Su}

Chinese Academy of Medical Sciences \& Peking Union Medical College Fuwai Hospital

\section{Li-Hong Ma ( $\nabla$ mlh8168@163.com )}

Chinese Academy of Medical Sciences \& Peking Union Medical College Fuwai Hospital

\section{Research}

Keywords: Percutaneous coronary intervention, coronary artery disease, major adverse cardiovascular event, integrative Chinese and western medicine.

Posted Date: November 23rd, 2021

DOI: https://doi.org/10.21203/rs.3.rs-1069902/v1

License: (9) This work is licensed under a Creative Commons Attribution 4.0 International License. Read Full License 


\section{Abstract}

Background Integrative Chinese and Western Medicine (ICWM) is widely used in coronary artery disease (CAD) patients after percutaneous coronary intervention (PCI) in China. However, the evidence-based on the long-term prognosis and large sample on this topic are weak. The purpose of this study is to evaluate the correlation between the therapeutic effect of ICWM and the prognosis of patients after PCI.

Methods This study is a prospective observational real-world cohort study that was conducted from September 2016 to August 2019 in Fuwai Hospital. The study was reviewed and approved by the Ethics Review Committee of Fuwai Hospital, Chinese Academy of Medical Sciences. We consecutively screened 6000 patients after $\mathrm{PCl}$ and they were followed up for 2 years. ICWM were related to prognostic outcomes using unadjusted (Kaplan-Meier curves) and risk-adjusted (multivariable Cox regression) analyses. The primary endpoint was a composite of all-cause death, revascularization, and myocardial infarction.

Results A total of 5942 patients after $\mathrm{PCl}$ were enrolled in this study, 5453 patients were included in the final analysis (4189[76.8\%] were male; mean [SD] age, 61.91[9.91] years). There were 2932 patients (53.8\%) in western medicine group (WMG) and 2521 patients (46.2\%) in integrated medicine group (IMG). Cox regression analysis showed that IMG had a $27 \%$ lower cumulative risk of the major adverse cardiovascular event (MACE) than WMG (hazard ratio [HR], 0.73; 95\% Cl, 0.63-0.85; P凶0.0001), especially in all-cause mortality and revascularization.

Conclusions Among patients after PCl, ICWM compared with conventional western medicine was correlated with a lower risk of 2-year MACE. Further research is needed to provide higher levels of evidence.

\section{Background}

Coronary artery disease (CAD) is a major public challenge and the leading cause of morbidity and mortality worldwide ${ }^{1}$. Percutaneous coronary intervention $(\mathrm{PCl})$ is the most common strategy for treating CAD. In 2018, 915256 patients underwent PCl in the Chinese mainland ${ }^{2}$, which increased $21.5 \%$ year over year in $2017^{3}$. With the development of interventional techniques, medical apparatus and instruments, the survival risk of patients with CAD is greatly reduced, clinicians and researchers begin to pay more attention to the postoperative cardiac rehabilitation of patients. $\mathrm{PCl}$ can achieve patients' coronary artery reperfusion, but $\mathrm{PCl}$ can lead to vascular endothelial injury as a kind of invasive operation, it cannot change the etiology and pathogenesis of CAD. Even if the patients after PCI take conventional western drugs (such as aspirin, clopidogrel, statins, etc.), there are still about $5-15 \%$ risk of major adverse cardiovascular event (MACE) within one year after $\mathrm{PCl}^{4-6}$. In addition, conventional western drugs cannot be used to some patients due to allergies or resistance ${ }^{7}$. Therefore, the secondary prophylactic drug strategy after $\mathrm{PCl}$ needs to be improved. 
Many CAD patients after PCl are treated with traditional Chinese medicine (TCM) based on conventional western medicine in China, and previous evidence-based studies on integrated Chinese and western medicine (ICWM) for CAD showed that it can reduce recurrence angina frequency ${ }^{8}$ and the occurrence of adverse cardiovascular events ${ }^{9-11}$, alleviate the clinical symptoms ${ }^{12}$ and the quality of life of patients ${ }^{13}$, ameliorating hemorheology and blood lipid parameters, inflammatory mediators ${ }^{14}$. But the sample size of these studies is small, and the follow-up time is short. On the other extreme, meta-analyses on the results have been conducted in ${ }^{14-16}$. Unfortunately, due to the inconsistency in the context and circumstances of these studies, further insight and consensus are to reach. Thus, the evidence for the role of TCM in long-term curative effects after PCl is not sufficient. Additionally, the evidence of real-world study about integrated Chinese and western medicine treatment for CAD is insufficient. Therefore, we designed this large sample, long-term follow up, real-world study with the following objectives: (1) to observe the treatment effects of Chinese patients after $\mathrm{PCl}$ in the real-world, (2) to identify the incidence and trends in MACE after $\mathrm{PCl}$, (3) to compare the risk of MACE within 2-year after $\mathrm{PCl}$ among western medicine group (WMG) and integrative medicine group (IMG).

\section{Methods}

\section{Study Design and Patients}

This study was a prospective observational real-world cohort study. During the period from enrollment to the end of follow-up, no intervention was carried out for patients. We observed patients' medication strategies and outcomes under real medical conditions in China. Follow-up was conducted on 3th, 6th, 12th, 18th, and 24th months after $\mathrm{PCl}$ (with a window of \pm 14 days). The study was reviewed and approved by the Ethics Review Committee of Fuwai Hospital, Chinese Academy of Medical Sciences.

Patients diagnosed with CAD after PCl within a week in Fuwai hospital Chinese Academy of Medical Sciences from September 2016 to August 2017 were included. Only stents were included, not balloon dilatation. Exclusion criteria include (1) age $\leq 18$ years old; (2) cognitive or communication impairment; (3) Participating in other clinical studies. All participants gave written informed consent.

\section{Groups and Medicine Strategies}

Patients were divided into WMG and IMG according to their medicine strategies during the 2-year followup. If the patients after PCl used western medicine in combination with TCM for more than half a year, they were divided into IMG; Otherwise, the patients were classified into WMG. Because the cohort distribution of patients was not determined before the end of the last follow-up, data on the lost patients were directly deleted.

Conventional western medicine refers to the routine medication for $C A D$ patients after $\mathrm{PCl}$, including aspirin, clopidogrel, statins, $\beta$-blocker, ACEI/ARB, etc. The specific strategy was determined by the clinician. TCM refers to the drug treatment based on syndrome differentiation, including decoction, powder and Chinese patent medicine. Decoction and powder are composed by many kinds of herbal 
drugs, such as Ren Shen, San Qi, Angelica Sinensis, et al., the specific drug types and doses were decided by clinicians according to the patients' TCM syndrome classification. Chinese patent medicines are certain forms according to the prescribed prescription and preparation technology, which were commercial TCM preparation approved by China Food and Drug Administration.

\section{Outcome Measures}

The primary endpoint was MACE, including all-cause mortality, myocardial infarction (MI), and revascularization, which was a composite of PCI, PTCA and CABG. The secondary endpoints were angina symptom score (ASS) and traditional Chinese medicine syndrome quantification score (TCMSS). The safety were evaluated by cardiovascular hospitalization and stroke. An independent endpoint committee adjudicated all potential events. All endpoint events of patients were evaluated by an independent endpoint committee.

\section{Sample size calculation}

In this study, it is expected that the data of 6,000 patients who were successively enrolled in Fuwai Hospital after $\mathrm{PCl}$ will be accumulated during the first year. According to previous experience, the proportion of patients treated with ICWM can reach 1/3 level in Fuwai Hospital. Combined with the literature ${ }^{17}$ and clinical data of our hospital, the incidence of primary endpoint events 2 years after $\mathrm{PCl}$ was about $15-20 \%$. According to this incidence level, the number of primary endpoints that can be accumulated by the end of the study is expected to 1200 . As an event-driven study, based on a bilateral significant level of $2.5 \%$ and an $80 \%$ degree of assurance, a statistically significant result can be obtained when a relative reduction of $20 \%(\mathrm{HR}=0.8)$ or more in the risk of the primary endpoint in IMG compared to WMG with the above sample size.

\section{Statistical Analysis}

Continuous variables are expressed in the form of mean and standard deviation (SD), categorical variables are described in the form of frequency and percentage. According to the distribution characteristics of data, the corresponding parameter or non-parameter statistical method is selected. Baseline characteristics were compared between two groups using T-tests or $\chi^{2}$ tests. The primary endpoint and the safety endpoints were analyzed by the survival analysis. In addition to describing the occurrence of events in each group with the Kaplan Meier curve, Cox proportional hazard model was used to estimate the hazard ratio (HR) and its $95 \%$ confidence interval $(\mathrm{Cl})$. To further explore the prognostic differences between the two groups, we selected some baseline characteristics as covariables for multivariate Cox regression analysis based on previous studies and clinical significance. The secondary endpoints were analyzed by T-tests. The significance level of the statistical test was $5 \%$ on two sides, and the statistical analysis software was intended to use SAS 9.4.

\section{Patient and Public Involvement statement}

During the initial enrollment phase, the patients were involved in this study as the subjects and signed informed consent. They were followed-up by telephone 2 years after enrollment. The patients/the public 
were not involved in the design or plan of this study, and they were not asked to assess the burden of the intervention because this is an observational study.

\section{Results}

\section{Study Patients and Baseline Characteristics}

We screened 6,000 patients after $\mathrm{PCl}, 5942$ of whom were included in this study. 5471 patients completed the 2-year follow-up, of which 5453 (4189 male [76.8\%]; mean [SD] age, 61.91 [9.91] years) were included in the final data analysis (Figure1). Among them, there were 2932 patients $(53.77 \%)$ in WMG and 2521 patients (46.23\%) in IMG. Baseline demographic and clinical characteristics of patients in two groups are described in Table1. In terms of demographic data, WMG is a younger, more male group compared to IMG. Regarding the severity of CAD, WMG had shorter duration, but more patients with a history of myocardial infarction than IMG.

\section{Primary Outcome}

All patients were followed up for about 2 years, 740 patients experienced MACE. The incidence of MACE was $15.31 \%$ (449/2932) in WMG and 11.54\% (291/2521) in IMG. IMG has a $27 \%$ lower cumulative risk of MACE than WMG (hazard ratio [HR], 0.73; 95\% Cl, 0.63-0.85; Pख0.0001) (Figure 2A). Based on previous studies and clinical significance, we selected some baseline characteristics as covariables for Cox regression analysis (age, gender, the course of CAD, history of $\mathrm{MI}, A C S$, first $\mathrm{PCl}$, overweight or obesity, current smoker, hypertension, hyperlipemia, diabetes). After adjusting these baseline characteristics, multivariate Cox regression analysis showed a significant difference between WMG and IMG (HR, 0.72; $95 \% \mathrm{Cl}, 0.62-0.84 ; \mathrm{P} \otimes 0.0001)$. Then, we excluded the patients with poor medication compliance during follow-up (12 patients in WMG and 33 patients in IMG), the results of multivariate Cox still showed that the risk of MACE was significantly lower in IMG than WMG (HR, 0.71; 95\% Cl, 0.61-0.82; Pه0.0001).

The incidence of the components of MACE were further investigated. The differences of two groups in allcause mortality ( $\mathrm{HR}, 0.11 ; 95 \% \mathrm{Cl}, 0.05-0.24 ; \mathrm{P}<0.0001)$ and revascularization (HR, $0.82 ; 95 \% \mathrm{Cl}, 0.70-0.96$; $\mathrm{P}=0.012$ ) were statistically significant (Figure $2 \mathrm{~B} \& \mathrm{C}$ ). The number of patients who experienced $\mathrm{MI}$ was so small that Cox regression analysis was not performed (6 patients in WMG vs. 3 patients in IMG).

\section{Secondary Outcomes}

After excluding 77 deaths during 2 years (69 patients in WMG and 8 patients in IMG), 5376 survival patients were followed up with ASS and TCMSS. We analyzed the difference value between the last follow-up and baseline. Table 2 showed that the variations of ASS $(P<0.001)$ and TCMSS $(P<0.001)$ were both significantly different between two groups.

\section{Safety Outcomes}

Figure 3 showed the cumulative risks of cardiovascular hospitalization and stroke between the two groups. 406 patients (13.85\%) in WMG and 296 patients (11.74\%) in IMG hospitalized for cardiovascular 
reasons during the 2-year follow up. Multivariate Cox regression analysis showed that the risk of cardiovascular hospitalization in IMG was lower 19.1\% than WMG (HR, 0.81; 95\% $\mathrm{Cl}, 0.70-0.94 ; \mathrm{P}=0.007)$. In terms of stroke, 24 patients $(0.82 \%)$ in WMG and 23 patients $(0.91 \%)$ in IMG experienced it. Multivariate Cox regression indicated that there was no significant difference in the incidence of stroke between the two groups $(\mathrm{HR}, 1.04 ; 95 \% \mathrm{Cl}, 0.58-1.86 ; \mathrm{P}=0.896)$.

\section{Discussion}

To our knowledge, this study is the largest prospective clinical study on the ICWM for CAD patients after $\mathrm{PCl}$ with MACE as the primary outcome index, and it is also the first study focusing on the real-world efficacy on this topic. The results demonstrated that compared to conventional western medicine alone, ICWM showed a significantly lower risk of MACE (especially in all-cause mortality and revascularization) and milder clinical symptoms.

Our study found that ICWM had a significant advantage in the prognosis of patients after PCl, which was consistent with the results of previous RCT studies. $5 \mathrm{C} \mathrm{trial}^{9}$ of 808 patients with acute coronary syndrome after $\mathrm{PCl}$ reported that ICWM can bring a significant benefit to MACE. Similarly, another study ${ }^{10}$ of 1054 patients with stable CAD showed that ICWM reduced the incidence of MACE. In terms of observational studies, a prospective cohort study of $334 \mathrm{AMI}$ patients showed that ICWM is one of the protective factors. ${ }^{18}$ Another prognostic analysis of 5284 patients with CAD got a similar result, which showed that ICWM has a potential role in reducing MACE, but there was a great difference in sample size between the two groups, which may reduce the statistical power. ${ }^{19}$ However, some of studies showed that ICWM can only benefit clinical symptom scores, but cannot reduce the rate of cardiovascular events ${ }^{12,13}$. The different results may be related to the sample size, follow-up time, drugs and definition of MACE.

Overall, the 2-year incidence of MACE in this study is $13.3 \%$ (15.0\% in WMG vs. $11.2 \%$ in IMG), which is consistent with the incidence in recent relevant studies ${ }^{20-23}$. However, this study showed a significant difference in mortality between two groups, which has an obvious discrepancy with previous studies about ICWM for patients after $\mathrm{PCl}^{8-10,13}$. The results differed from the previous studies may be caused by the following reasons: firstly, the sample size of those studies was small and the follow-up time was short. Secondly, most of the previous studies focused on cardiovascular mortality, rather than not allcause mortality. TCM is not only aimed at the heart or coronary artery but regards the patient as a whole, many patients in this study have other life-threatening diseases such as cancers, heart failure, abnormal liver or kidney function, or other complications. The holistic view of Chinese medicine may be beneficial to the prognosis of patients. Thirdly, the medication and endpoint events information for the post-mortem follow-up of the deceased patient was obtained from the family, rather than from themselves. This problem may lead to deviation in the classification of the deceased, but this is inevitable. In addition, we asked multiple family members of each patient to reduce their memory bias.

In this study, the actual clinical medication of patients within two years after PCI was recorded. The TCM taken by the patients in this study has been extensively utilized in clinical practice in China, some of 
which have been proved to have a significant effect on CAD in previous clinical research ${ }^{8,14,24}$. In terms of the mechanism of drug action, the previous studies showed that Shexiang Baoxin Pill can reduce myocardial infarction area and protect cardiac function through promoting angiogenesis ${ }^{25}$. Qishen capsules can increase angiogenesis to improve the cardiac function of rats after $\mathrm{Ml}$ by the Akt signaling pathway ${ }^{26}$. Tongxinluo can alleviate myocardial ischemia/reperfusion injury by activating cardiac microvascular endothelial cells endothelial nitric oxide synthase ${ }^{27}$. Compound danshen dripping pills ameliorated myocardial ischemia, reversed the reprogramming of the metabolism induced by ISO and normalized the level of most myocardial substrates and the genes/enzymes associated with those metabolic changes ${ }^{28}$.

On the one hand, this approach reflects the effect of ICWM in clinical practice, where the TCM may not be continually taken, thus evaluating the effect of ICWM in a more practical and comprehensive manner. On the other hand, the difficulties of standardizing the intervention factors in TCM treatment and drugs, which may vary according to syndromes in response to time, place, climate etc. beyond the disease in TCMs are avoided.

Notably, this study, based on real-world data, can help to augment and extrapolate data obtained in randomized controlled trials and provide information about the safety and effectiveness of the medication in heterogeneous and large populations. This is an attempt to explore and evaluate the efficacy of ICWM based on the changeable clinical characteristics of TCM application. The patients usually take TCM only when they feel uncomfortable in China, few patients take TCM continually as they did in RCTs. Therefore, compared to the previous RCTs, this study can better reflect the clinical practice of ICWM in the treatment of CAD in the real world. In addition, the disease types of patients with CAD are the same, but the syndromes of them are different, and dialectical treatment is the fundamental principle of TCM, so the drugs in the sight of TCM are also different for them. Furthermore, the syndromes of patients will change with time, place, climate and other factors, TCM also needs to be adjusted accordingly. As a result, on the one hand, all patients with CAD using the same TCM are not in line with the theoretical basis of TCM and clinical usage of the actual situation. On the other hand, RCTs need to accurately define the intervention, to determine the impact of a specific intervention factor on the study endpoints. Two of them are contradictory and it is one of the difficulties in the development of evidence-based studies for TCM. So, we try to use this type of study to discuss this issue.

This study has the following limitations: firstly, as a prospective, observational, real-world study, inclusion criteria were wider than the RCT study, and patient heterogeneity was greater. Although multivariate adjustment was performed in Cox analysis of endpoint events, other unknown factors that may have influenced the results may still exist; secondly, this study is single-center clinical research, which may limit the generalizability of the results, although it may promote consistency of treatment and evaluation. We are looking forward to relevant RCTs with a large sample size and long follow-up time to verify our study.

As for the cohort study, the main shortage was bias and confounding factors, which influences the reliability of the study. In the endpoint events analysis present study, we adjusted for baseline 
characteristics that might affect prognosis to make a good match between two groups, such as demographic characteristics, disease history, and comorbidities. Moreover, the losing follow-up rate is only $4.0 \%$ in the present study. Therefore, it is reasonable to believe that the present clinical trial had a well-controlled confounding bias, which indicated the results had good reliability and credibility.

\section{Conclusions}

Compared to western medicine alone, ICWM had a significant lower risk of 2-year MACE among the patients after $\mathrm{PCl}$, especially in terms of all-cause mortality and revascularization. The additional benefits of TCM based on conventional western medicines can be anticipated to further improve the second prevention of CAD in the future.

\section{Abbreviations}

ICWM

integrated Chinese and Western medicine

CAD

coronary artery disease

$\mathrm{PCl}$

percutaneous coronary intervention

WMG

western medicine group

IMG

integrative medicine group

MACE

major adverse cardiovascular event

TCM

traditional Chinese medicine

MI

myocardial infarction

ASS

angina symptom score

TCMSS

traditional Chinese medicine syndrome quantification score

HR

hazard ratio

$\mathrm{Cl}$

confidence interval

RCT

randomized controlled trial 


\section{Declarations}

Ethics approval and consent to participate: The study was reviewed and approved by the Ethics Review Committee of Fuwai Hospital, Chinese Academy of Medical Sciences (Approval NO. 2016-786). All participants gave written informed consent.

Consent for publication: All the authors agree to publication in the journal.

Availability of data and materials: The datasets used or analysed during the current study are available from the corresponding author on reasonable request.

Competing interests: The authors declare that they have no competing interests.

Funding: This work was supported by the Capital Health Development Project of China grant [grant numbers SHF-2016-2-4032].

Authors' contributions: Li-Hong Ma and Si-Yu Yan had full access to all of the data in the study and take responsibility for the integrity of the data and the accuracy of the data analysis. Concept and design: LiHong Ma, Wei-Xian Yang, Cai-Xia Guo, Pei-Pei Lu. Acquisition, analysis, or interpretation of data: Si-Yu Yan, Pei-Pei Lu, Xuan-Tong Guo, Si-Yu Yan. Drafting of the manuscript: Si-Yu Yan, Pei-Pei Lu, Li-Hong Ma. Critical revision of the manuscript for important intellectual content: Li-Hong Ma. Obtained funding: LiHong Ma. All of the authors have read and approved the submitted manuscript.

\section{Acknowledgments}

We thank Medical Statistics Division of Fuwai Hospital, Chinese Academy of Medical Sciences for data analysis. We thank Beijing Econ Medical Technology for the construction and maintenance of the data collection platform. We thank all patients who participated in this study.

\section{References}

1. Sanchis-Gomar F, Perez-Quilis C, Leischik R and Lucia A. Epidemiology of coronary heart disease and acute coronary syndrome. Annals of translational medicine. 2016;4:256.

2. Report on cardiovascular health and disease in China 2019. Journal of Cardiovascular and Pulmonary Diseases. 39(09):1145-1156.

3. Sheng-shou HU, Run-lin GAO, Li-sheng LIU, et al. Synopsis of China cardiovascular disease report 2018. Chinese Circulation Journal. 2019,34(03):209-220.

4. Varenne O, Cook S, Sideris G, Kedev S, Cuisset T, Carrié D, Hovasse T, Garot P, El Mahmoud R, Spaulding C, Helft G, Diaz Fernandez JF, Brugaletta S, Pinar-Bermudez E, Mauri Ferre J, Commeau P, Teiger E, Bogaerts K, Sabate M, Morice M-C and Sinnaeve PR. Drug-eluting stents in elderly patients with coronary artery disease (SENIOR): a randomised single-blind trial. The Lancet. 2018;391:41-50. 
5. Fanaroff AC, Zakroysky P, Wojdyla D, Kaltenbach LA, Sherwood MW, Roe MT, Wang TY, Peterson ED, Gurm HS, Cohen MG, Messenger JC and Rao SV. Relationship Between Operator Volume and LongTerm Outcomes After Percutaneous Coronary Intervention. Circulation. 2019;139:458-472.

6. Madhavan MV, Kirtane AJ, Redfors B, Genereux P, Ben-Yehuda O, Palmerini T, Benedetto U, BiondiZoccai G, Smits PC, von Birgelen C, Mehran R, McAndrew T, Serruys PW, Leon MB, Pocock SJ and Stone GW. Stent-Related Adverse Events $>1$ Year After Percutaneous Coronary Intervention. J Am Coll Cardiol. 2020;75:590-604.

7. Latib A, lelasi A, Ferri L, Chieffo A, Godino C, Carlino M, Montorfano M and Colombo A. Aspirin intolerance and the need for dual antiplatelet therapy after stent implantation: a proposed alternative regimen. International journal of cardiology. 2013;165:444-7.

8. Ge JB, Fan WH, Zhou JM, Shi HM, Ji FS, Wu Y, Zhao YL, Qian J, Jin YZ, Liu YW, Wang SH, He SH, Yang P, Wu J, Lu F and Hou ZS. Efficacy and safety of Shexiang Baoxin pill (MUSKARDIA) in patients with stable coronary artery disease: a multicenter, double-blind, placebo-controlled phase IV randomized clinical trial. Chinese medical journal. 2020;134:185-192.

9. Wang SL, Wang CL, Wang PL, Xu H, Liu HY, Du JP, Zhang DW, Gao ZY, Zhang L, Fu CG, Lü SZ, You SJ, Ge JB, Li TC, Wang X, Yang GL, Liu HX, Mao JY, Li RJ, Chen LD, Lu S, Shi DZ and Chen KJ. Combination of Chinese Herbal Medicines and Conventional Treatment versus Conventional Treatment Alone in Patients with Acute Coronary Syndrome after Percutaneous Coronary Intervention (5C Trial): An Open-Label Randomized Controlled, Multicenter Study. Evidence-based complementary and alternative medicine : eCAM. 2013;2013:741518.

10. Guo M, Wang P, Du J, Fu C, Yang Q, Gao Z, Zhu M, Lv S, Deng Y, Li T, Shi D and Working Group FTX. Xinyue Capsule in patients with stable coronary artery disease after percutaneous coronary intervention: a multicenter, randomized, placebo-controlled trial. Pharmacological research. 2020;158:104883.

11. Wang L, Zhao X, Mao S, Liu S, Guo X, Guo L, Du T, Yang H, Zhao F, Wu K, Cong H, Wu Y, Yang PC, Chen $\mathrm{K}$ and Zhang M. Efficacy of Danlou Tablet in Patients with Non-ST Elevation Acute Coronary Syndrome Undergoing Percutaneous Coronary Intervention: Results from a Multicentre, PlaceboControlled, Randomized Trial. Evidence-based complementary and alternative medicine : eCAM. 2016;2016:7960503.

12. Zhao S, Tang Y, Cai H, Liu W, Zhang L, Chen D and Chen B. Treatment of Danhong Injection Combined with Naoxintong Capsule in Acute Coronary Syndrome Patients Undergoing PCI Operation: Study for a Randomized Controlled and Double-Blind Trial. Evidence-based complementary and alternative medicine : eCAM. 2018;2018:8485472.

13. Wu HL, Wang YF, Li JZ, Zhang MZ, Sheng XG, Wang X, Li S, Chen QX, Li XQ, Ou AH and Ruan XM. A multicentre randomized clinical trial on efficacy and safety of huxin formula in patients undergoing percutaneous coronary intervention. Evidence-based complementary and alternative medicine : eCAM. 2014;2014:143064. 
14. Li C, Li Q, Xu J, Wu W, Wu Y, Xie J and Yang X. The Efficacy and Safety of Compound Danshen Dripping Pill Combined with Percutaneous Coronary Intervention for Coronary Heart Disease. Evidence-based complementary and alternative medicine : eCAM. 2020;2020:5067137.

15. Zheng GH, Liu JP, Chu JF, Mei L and Chen HY. Xiongshao for restenosis after percutaneous coronary intervention in patients with coronary heart disease. Cochrane Database of Systematic Reviews. 2013.

16. Mao C, Fu XH, Yuan JQ, Yang ZY, Chung VC, Qin Y, Huang Y, Tam WW, Kwong JS, Xie W and Tang JL. Tong-xin-luo capsule for patients with coronary heart disease after percutaneous coronary intervention. The Cochrane database of systematic reviews. 2015:Cd010237.

17. Chinese expert consensus on antiplatelet therapy. Chinese Journal of Cardiology. 2013(03):183-194.

18. Duan WH, Lu F, Li LZ, Wang CL, Liu JG, Yang QN, Gu F, Zhang L and Shi DZ. Clinical efficacy of traditional chinese medicine on acute myocardial infarction: a prospective cohort study. Chinese journal of integrative medicine. 2012;18:807-12.

19. Gao ZY, Xu H, Shi DZ, Wen C and Liu BY. Analysis on outcome of 5284 patients with coronary artery disease: the role of integrative medicine. Journal of ethnopharmacology. 2012;141:578-83.

20. Yamamoto MH, Maehara A, Stone GW, Kini AS, Brilakis ES, Rizik DG, Shunk K, Powers ER, Tobis JM, Maini BS, Dixon SR, Goldstein JA, Petersen JL, 2nd, Généreux P, Shah PR, Crowley A, Nicholls SJ, Mintz GS, Muller JE and Weisz G. 2-Year Outcomes After Stenting of Lipid-Rich and Nonrich Coronary Plaques. Journal of the American College of Cardiology. 2020;75:1371-1382.

21. Rha SW, Choi BG, Choi SY, Choi CU, Gwon HC, Chae IH, Kim HS, Park HS, Lee SH, Kim MH, Hur SH and Jang Y. Multicenter experience with percutaneous coronary intervention for chronic total occlusion in Korean population: analysis of the Korean nationwide multicenter chronic total occlusion registry. Coronary artery disease. 2020;31:319-326.

22. Plitt A, Claessen BE, Sartori S, Baber U, Chandrasekhar J, Aquino M, Vijay P, Elsayed S, Kovacic JC, Sweeny J, Barman N, Moreno P, Krishnan P, Demopoulos A, Dangas G, Kini AS, Mehran R and Sharma SK. Impact of stent diameter on outcomes following percutaneous coronary intervention with second-generation drug-eluting stents: Results from a large single-center registry. Catheterization and cardiovascular interventions : official journal of the Society for Cardiac Angiography \& Interventions. 2020;96:558-564.

23. Beyhoff N, Cao D, Mehran R, Dangas G, Baber U, Sartori S, Blum M, Roumeliotis A, Chandiramani R, Goel R, Zhang Z, Kovacic J, Krishnan P, Barman N, Kapur V, Sweeny J, Sharma SK and Kini A. Prognostic Impact of High-Sensitivity C-Reactive Protein in Patients Undergoing Percutaneous Coronary Intervention According to BMI. JACC Cardiovascular interventions. 2020;13:2882-2892.

24. Zeng Z, Zhuang Z, He Y, Yang Z and Song Y. Efficacy and safety of Shen-Song-Yang-Xin capsule for treating arrhythmia in the elderly patients with coronary heart disease: Protocol for a systematic review and meta-analysis. Medicine. 2018;97:e13599.

25. Zhang KJ, Zhu JZ, Bao XY, Zheng Q, Zheng GQ and Wang Y. Shexiang Baoxin Pills for Coronary Heart Disease in Animal Models: Preclinical Evidence and Promoting Angiogenesis Mechanism. 
Frontiers in pharmacology. 2017;8:404.

26. Guo CX, Li ZY, Niu JB, Fan SC, Yan SY, Lu PP, Su YN and Ma LH. Qishen capsule safely boosts cardiac function and angiogenesis via the MEK/ERK pathway in a rat myocardial infarction model. Journal of geriatric cardiology : JGC. 2019;16:764-774.

27. Chen G, Xu C, Gillette TG, Huang T, Huang P, Li Q, Li X, Li Q, Ning Y, Tang R, Huang C, Xiong Y, Tian X, Xu J, Xu J, Chang L, Wei C, Jin C, Hill JA and Yang Y. Cardiomyocyte-derived small extracellular vesicles can signal eNOS activation in cardiac microvascular endothelial cells to protect against Ischemia/Reperfusion injury. Theranostics. 2020;10:11754-11774.

28. Aa N, Guo JH, Cao B, Sun RB, Ma XH, Chu Y, Zhou SP, Aa JY, Yang ZJ, Sun H and Wang GJ. Compound danshen dripping pills normalize a reprogrammed metabolism of myocardial ischemia rats to interpret its time-dependent efficacy in clinic trials: a metabolomic study. Metabolomics : Official journal of the Metabolomic Society. 2019;15:128.

\section{Tables}

Table1. Baseline demographic and clinical characteristics of patients 
Characteristics

Demographics

\begin{tabular}{|c|c|c|}
\hline Age, mean (SD), $\mathrm{y}^{*}$ & $61.59(9.92)$ & $62.28(9.88)$ \\
\hline Men, No. $(\%)^{*}$ & $2312(78.9)$ & $1877(74.5)$ \\
\hline Height, mean (SD), $\mathrm{cm}^{*}$ & $168.53(7.08)$ & $168.08(7.36)$ \\
\hline Weight, mean (SD), Kg & $73.64(11.12)$ & $73.14(11.07)$ \\
\hline $\mathrm{BMI}$, mean (SD), $\mathrm{Kg} / \mathrm{m}^{2}$ & $25.86(3.08)$ & $25.81(3.00)$ \\
\hline \multicolumn{3}{|l|}{ Information about PCI } \\
\hline The course of CAD, mean (SD), $y^{\star}$ & $1.98(3.86)$ & $2.19(3.92)$ \\
\hline History of myocardial infarction, No. (\%) & $1103(37.6)$ & $827(32.8)$ \\
\hline ACS, No. (\%) & $1822(62.1)$ & $1611(63.9)$ \\
\hline First PCl, No. (\%) & $1907(65.0)$ & $1581(62.7)$ \\
\hline \multicolumn{3}{|l|}{ Cardiac risk factors } \\
\hline Overweight or obesity, No. (\%) & $1759(60.0)$ & $1502(59.6)$ \\
\hline Current smoker, No. $(\%)^{*}$ & $1848(63.0)$ & $1453(57.6)$ \\
\hline hypertension, No. (\%) & $1938(66.1)$ & $1724(68.4)$ \\
\hline hyperlipemia, No. (\%) & $2617(89.3)$ & $2263(89.8)$ \\
\hline diabetes, No. (\%) & $1022(34.9)$ & $918(36.4)$ \\
\hline Family history of CAD, No. $(\%)^{*}$ & $545(18.6)$ & $596(23.6)$ \\
\hline \multicolumn{3}{|l|}{ Conventional western drugs } \\
\hline DAPT, No. $(\%)^{*}$ & $2910(99.2)$ & $2512(99.6)$ \\
\hline Nitrates, No. (\%) & $2744(93.6)$ & $2356(93.5)$ \\
\hline$\beta$-blocker, No. (\%) & $2566(87.5)$ & $2164(85.8)$ \\
\hline CCB, No. $(\%)^{*}$ & $1628(55.5)$ & $1513(60.0)$ \\
\hline ACEI/ARB, No. (\%) & $1782(60.8)$ & $1489(59.1)$ \\
\hline Statin, No. (\%) & $2878(98.2)$ & $2485(98.6)$ \\
\hline Diuretic, No. (\%) & $384(13.1)$ & $296(11.7)$ \\
\hline Antidiabetic, No. (\%) & $778(26.5)$ & $692(27.4)$ \\
\hline
\end{tabular}

WMG $(n=2932) \quad$ IMG $(n=2521)$

Demographics


Abbreviations: BMI, Body Mass Index; CAD, Coronary Artery Disease; ACS, Acute Coronary Syndrome; PCI, Percutaneous Coronary Intervention; DAPT, Dual Anti-Platelet Therapy; CCB, Calcium Channel Blockers; ACEI,

Angiotensin Converting Enzyme Inhibitor.

$* P<0.05$

\section{Table2. The variations of ASS and TCMSS of two groups}

\begin{tabular}{|c|c|c|c|c|}
\hline Scale & WMG $(n=2863)$ & IMG $(n=2514)$ & Statistics & $P$ value \\
\hline \multicolumn{5}{|l|}{ ASS $^{a}$} \\
\hline Mean \pm SD & $0.03 \pm 0.54$ & $-0.07 \pm 0.44$ & 7.773 & $<0.001$ \\
\hline Median (Q1, Q3) & $0.00 \rrbracket 0.00,0.00 \rrbracket$ & $0.00 \otimes 0.00,0.00 \rrbracket$ & & \\
\hline Minimum, Maximum & $-2.00,16.00$ & $-2.00,2.00$ & & \\
\hline \multicolumn{5}{|l|}{ TCMSS $^{b}$} \\
\hline Mean \pm SD & $0.22 \pm 1.04$ & $-0.59 \pm 1.15$ & 26.873 & $<0.001$ \\
\hline Median (Q1, Q3) & $0.00 \rrbracket 0.00 \rrbracket 0.00 \rrbracket$ & $-1.00 \rrbracket-1.00 \rrbracket 0.00 \rrbracket$ & & \\
\hline Minimum, Maximum & $-4.00 \rrbracket 8.00$ & $-6.00 \otimes 7.00$ & & \\
\hline
\end{tabular}

${ }^{a}$ ASS, Angina Symptom Score, consists of 4 items with a total score ranging from 0 to 24 , with higher scores

indicating more severe angina symptoms. It's the difference value between the last follow-up and baseline.

b TCMSS, Traditional Chinese Medicine Syndrome Quantification Score, consists of 5 items with a total score

ranging from 0 to 19, with higher scores indicating more severe syndrome of qi stagnation and blood stasis. It's the

difference value between the last follow-up and baseline. 


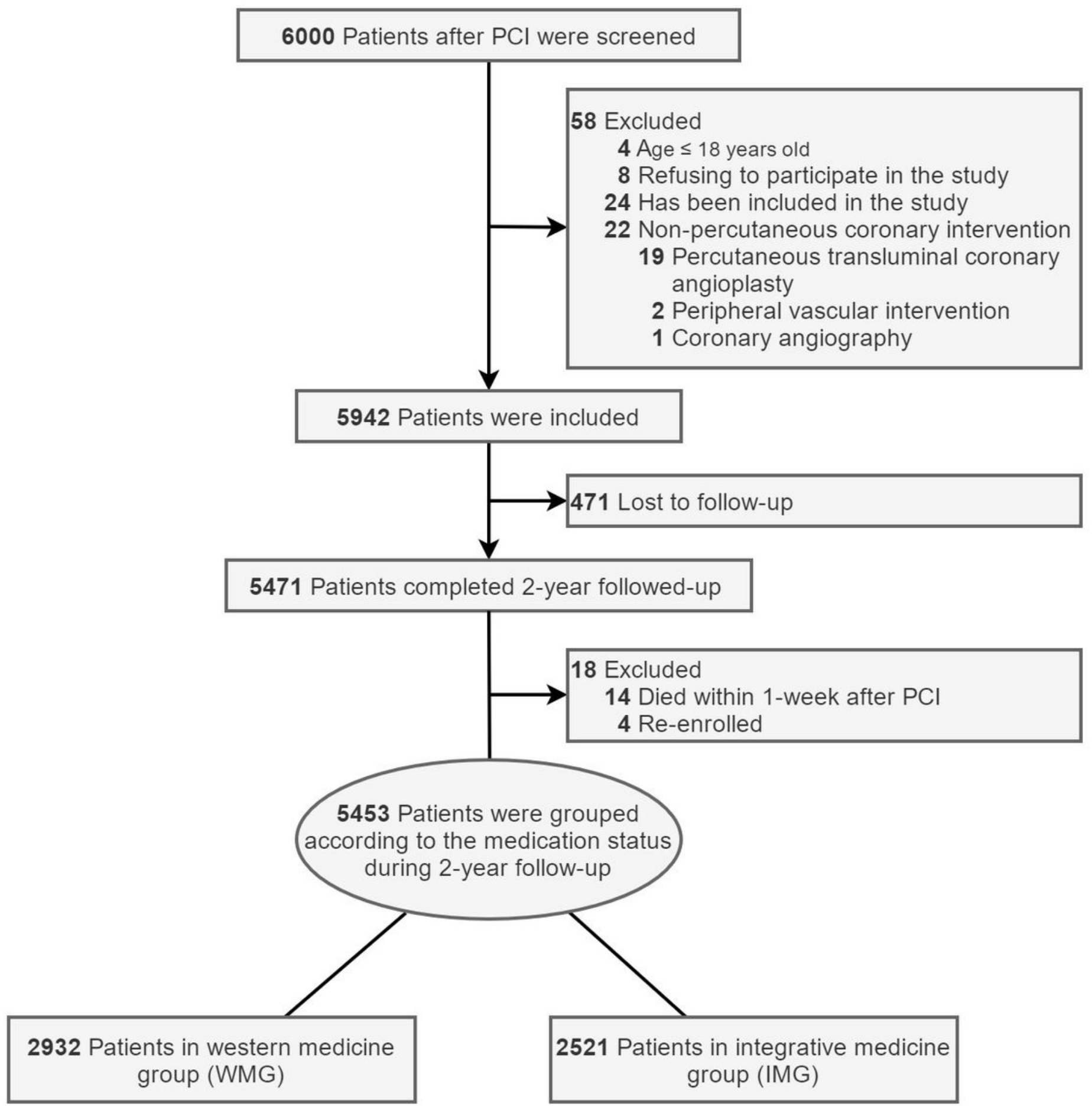

\section{Figure 1}

Study Flowchart of Participants Figure legends: PCI indicates percutaneous coronary intervention. 
A
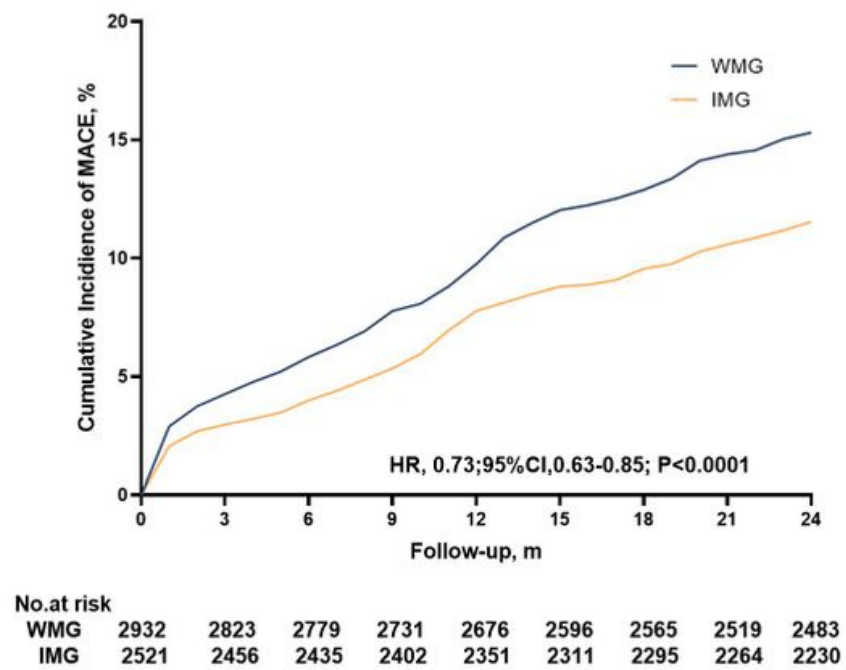

B

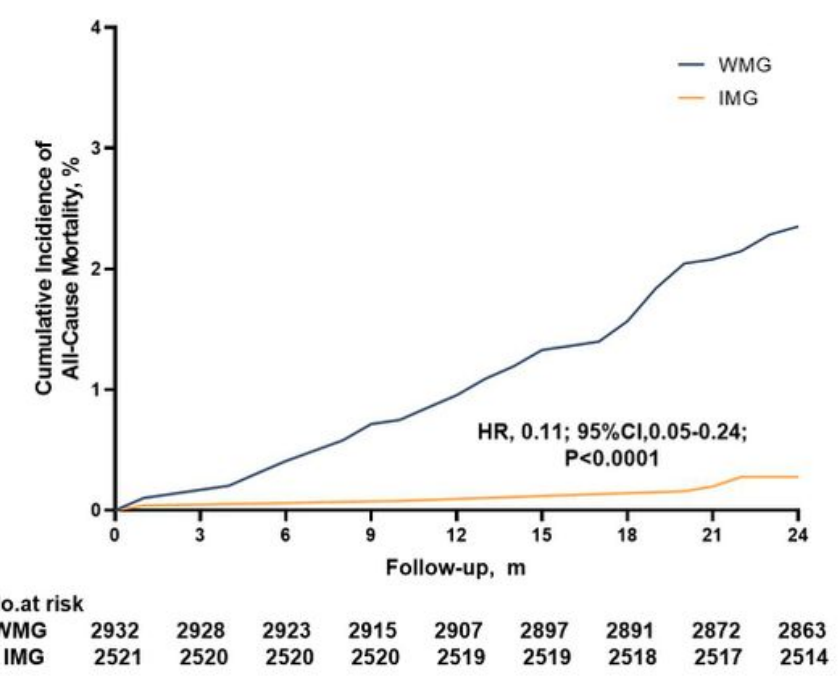

C

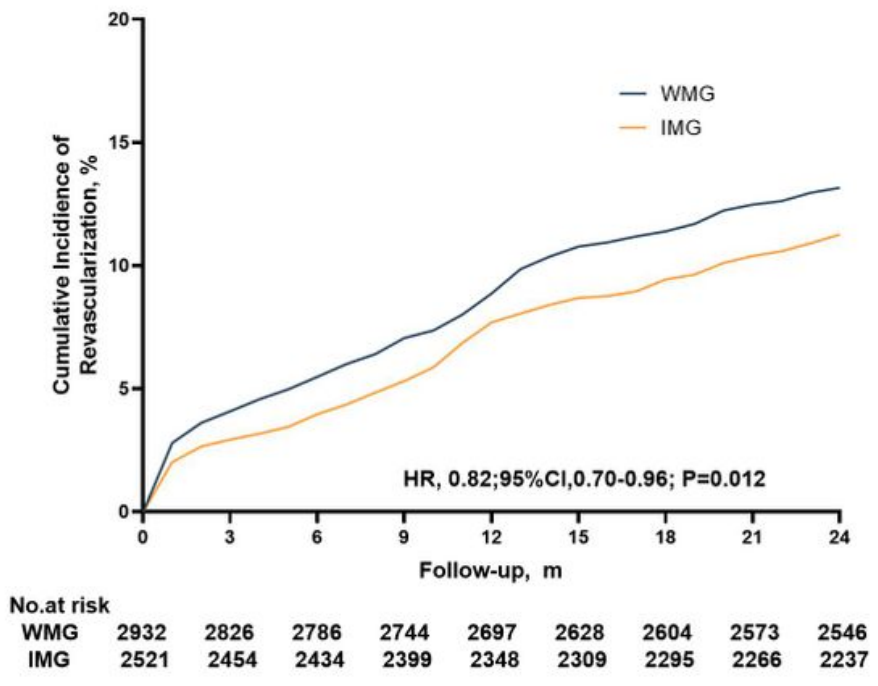

Figure 2

2-year Cumulative Incidence of the Primary Outcomes and the Components of MACE Among 5453 Patients after $\mathrm{PCl}$ 
A

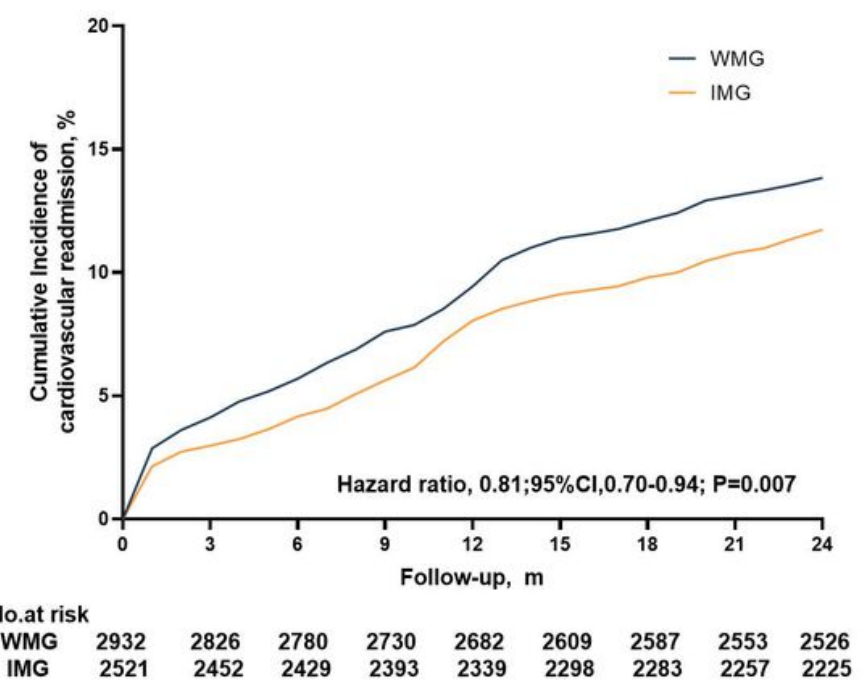

B

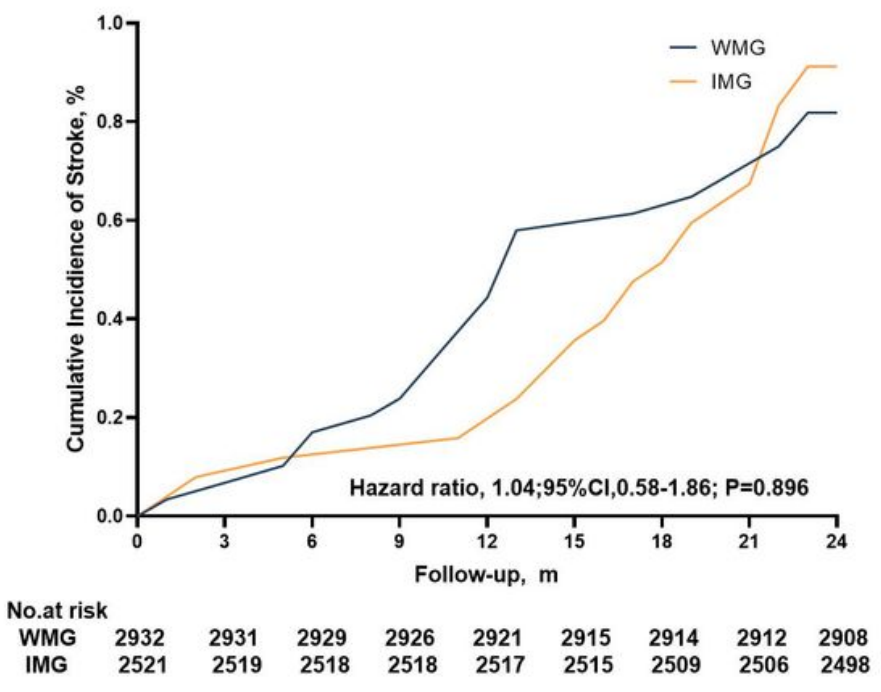

\section{Figure 3}

2-year Cumulative Incidence of the Safety Outcomes among 5453 Patients after PCI 